\title{
Erratum
}

\section{AVOIDING RISK DILEMMAS USING BACKCASTING}

\section{Mark Jablonowski}

Risk Management (2007) 9, 207

doi:10.1057/palgrave.rm.8250033

Correction to: Risk Management (2007) 9(2), 118-127, doi:10.1057/palgrave.rm.8250026

The abstract appearing on p. 118 of the article featured an error. The second sentence of the abstract was incorrectly given as:

"Precaution in the form of sensible alternatives assessment can help eliminate these dilemmas."

The above text should have appeared as follows:

"Preaction in the form of sensible alternatives assessment can help eliminate these dilemmas." 